\title{
IMPORTÂNCIA DOS ANIMAIS SINANTRÓPICOS NO CONTROLE DA ENDEMIA CHAGÁSICA *
}

Eduardo Olavo da Rocha e Silva** José Carlos Rehder de Andrade*** Anísio Ribeiro de Lima***

RSPU-B/276

\begin{abstract}
Rocha E SILVA, E. O. et al. - Importância dos animais sinantrópicos no controle da endemia chagásica. Rev. Saúde públ., S. Paulo, 9:371-81, 1975.

RESUMo: Foi salientado que, em certas areas, os métodos de rotina no controle dos vetores da doença de Chagas necessitam ser complementados por atividades de captura de resrevatórios do $\mathrm{T}$. cruzi, especialmente no caso, marsupiais (Didelphis) e roedores (Rattus). Foram citados trabalhos realizados em alguns municípios da Regiäo Administrativa 5 - Campinas, Estado de São Paulo, Brasil, onde a presença de reservatórios e triatomineos semi-domiciliarios (Panstrongylus megistus), nas moradias, propiciam condições à reintrodução do ciclo domiciliar do parasita. São comentados os resultados da pesquisa do Trypanosoma tipo cruzi, nesses triatomíneos e nos citados vertebrados, relacionando ainda os resultados com as reacōes de precipitina realizadas no sentido de detectar o tipo de sangue ingerido pelos P. megistus capturados.
\end{abstract}

UnITERmos: Tripanossomíase americana, epidemiologia. Panstrongylus megistus. Trypanosoma cruzi. Reservatórios.

\section{INTRODUCAO}

É fato já sobejamente conhecido que alguns marsupiais na natureza funcionam como reservatórios do Trypanosoma cruzi. Em conseqüência, procura-se hoje esclarecer o papel que eles exercem, notadamente os exemplares do gênero Didelphis, na manutenção e no inter-relacionamento das diversas cadeias de transmissão da doença de Chagas, ou seja, nos ciclos: silvestre, domiciliar e peridomiciliar da zoonose.

No momento, além da constatação da prevalência da infecção nos didelfídeos, interessam conhecimentos sobre aspectos da biologia e hábitos, bem como as implicações resultantes do comportamento dos triatomíneos encontrados nas proximidades dos locais de captura desses marsupiais, e as observações referentes à distância que o homem se encontra dos possiveis focos (Zeledon ${ }^{9}, 1974$ ).

Assunto correlato, também de grande interesse, refere-se à identificação correta dos tripanossomos observados, pois segundo Barreto e col. ${ }^{1}$ (1964), o simples encontro de formas flageladas semelhantes ao

* Trabalho apresentado no XI Congresso da sociedade Brasileira de Medicina Tropical, Rio de Janeiro, 23 a 28 de fevereiro de 1975.

* Do Departamento de Epidemiologia da Faculdade de Saúde Pública da USP - Avenida Dr. Arnaldo, 715 - São Paulo, SP - Brasil.

*** Superintendência de Controle de Endemias - Rua Tamandaré, 649 - São Paulo, SP - Brasil. 
ROCHA E SILVA, E. O. et al. - Importância dos animais sinantrópicos no controle da endemia chagásica Rer. Saúde públ., S. Paulo, 9:371-81, 1975

T. cruzi, em gotas e/ou esfregaços de sangue, ou o achado de tripanossomos no intestino posterior dos triatomíneos utilizados nos xenodiagnósticos, não constituem por si só elementos suficientes para caracterizar o agente da doença de Chagas.

$\mathrm{Na}$ identificação dos tripanossomos semelhantes ao $T$. cruzi encontrados em mamíferos. Barreto ${ }^{2}$ (1965) leva em consideração uma série de elementos que devem ser apreciados em conjunto. $O$ trabalho de Ribeiro e Juarez ' (1972) na identificação da infecção chagásica em um Oryzomys migripes, é bom exemplo do emprego integral das normas sugeridas por Barreto. A identificação dos tripanossomos, resultantes da captura de dezenas de animais, apresenta, evidentemente, implicaçôes outras de natureza operacional e de custo. Sendo assim, o próprio Barreto e col. ${ }^{1}$ (1964), ao capturar centenas de exemplares de gambás, não puderam observar nas amostras isoladas todos os itens recomendados.

Forattini e col. ${ }^{4}$ (1971), estudando a distribuição e dispersão dos triatomíneos em ecótopos naturais e artificiais, caracterizaram como $T$. cruzi os tripanossomos metacíclicos encontrados no tubo intestinal de triatomíneos que em laboratório infectaram camundongos jovens. Consideraram também como $T$. cruzi os tripanossomos metacíclicos que foram isolados através de xenodiagnóstico, do sangue de mamíferos. domésticos ou não. Sem dúvida mais completos, são os estudos que se fazem acompanhar de observaçóes sobre a presença de ninhos de formas tissulares (amastigotas), nos tecidos e órgãos. Foi este, aliás, o critério adotado pelo próprio Forattini e col. ${ }^{3}$ (1970), quando da notificação do primeiro achado do $T$. cru$z i$, no rato de esgoto (Rattus novergicus) e gambás (Didelphis marsupialis) na cidade de São Paulo.

\section{MATERIAL E MÉTODOS}

O material objeto deste trabalho resultou de capturas de vertebrados não domésticos, realizadas pelas equipes de campo da Diretoria de Combate a Vetores (SLSAM), no decorrer de atividades rotineiras de procura dos triatomíneos, em municípios da área sob controle do Serviço Regional-5 - Campinas, Estado de São Paulo.

Marsupiais e roedores foram capturados, alguns no interior de casas habitadas ou não, outros em dependências anexas às moradias e outros ainda em focos extradomiciliares periféricos às habitações.

Encaminhados ao laboratório de MojiGuaçu, esses animais eram de imediato identificados, registrados e examinados através de coleta de sangue, em preparações a fresco e coradas (Giemsa). A seguir, independentemente do resultado dos exames, eram submetidos ao xenodiagnóstico, empregando-se para tanto dez exemplares do Triatoma infestans, ninfas do $4 .^{\circ}$ e $/$ ou $5 .^{\circ}$ estádio, procedentes do insetário anexo ao referido laboratório.

Trinta e cinquienta dias após a realização dos xenodiagnósticos, os triatomíneos eram examinados. Apresentando o material coletado do tubo intestinal, formas flageladas, este era obrigatoriamente fixado com soro inativado e corado pelo MayGrunwald-Giemsa. Para melhor identificação, parcela do material intestinal, quando possível, era inoculada em camundongos jovens, semanalmente examinados até a quinta semana.

Os triatomíneos capturados pelas equipes, na medida em que chegavam ao laboratório, eram examinados de acordo com a técnica mencionada.

Na preparação de anti-soros e nas reações de precipitina (método dos tubos capilares), visando a identificação do material ingerido pelos triatomíneos, adotouse a técnica de Siqueira ${ }^{\circledR}(1960)$ com ligeiras modificações. 
ROCHA E SILVA, E. O. et al. - Importância dos animais sinantrópicos no controle da endemia chagásica Rev. Saúde públ., S. Paulo, 9:371-81, 1975.

\section{RESUL TA DOS}

Dos vertebrados xenados. intersesam sobretudo os gambás do gênero Didelphis e entre estes, pelo seu significativo número, o Didelphis azarae, cuja procedência e positividade para Trypanosoma tipo cruzi, mostramos na Figura e Tabela 1. Os resultados alcançados nas inoculações de camundongos, por via intraperitonial, das cepas isoladas através do xenodiagnóstico de alguns desses marsupiais, são apresentados na Tabela 2.

No que se refere a roedores do gênero Rattus, capturados em menor número, devido entre outros fatores, a maior dificuldade em apanhá-los nas condições de nosso experimento, os dados de procedência e infecção encontram-se na Tabela 3 .

A Tabela 4 mostra os resultados das capturas do $P$. megistus, realizadas nos anos de 1972 a 1974, nos municípios onde foram encontrados didelfídeos infectados pelo $T$. tipo cruzi.
Nas Tabelas 5 e 6 são apresentados os resultados dos testes de precipitina, realizados com material do tubo intestinal dos triatomíneos, procurando estabelecer através do conhecimento do tipo de sangue ingerido pelos $P$. megistus, suas relações com os reservatórios estudados. Na Tabela 5, os resultados dos exames foram distribuídos de acordo com os locais de captura dos exemplares e positividade para a bateria de anti-soros disponível: homem, cão, ave, roedor e gambá. Na Tabela 6 procurou-se estabelecer relação entre: fonte de alimento, estádio evolutivo do exemplar e infecção ou não pelo $T$. tipo cruzi.

\section{COMENTARIOS}

Pelos resultados alcançados por Barreto e col. ${ }^{1}$ (1964) - devido ao grande número de exemplares de $D$, azarae examinados, e também por terem sido captura-

ESTADO DE SÃO PAULO

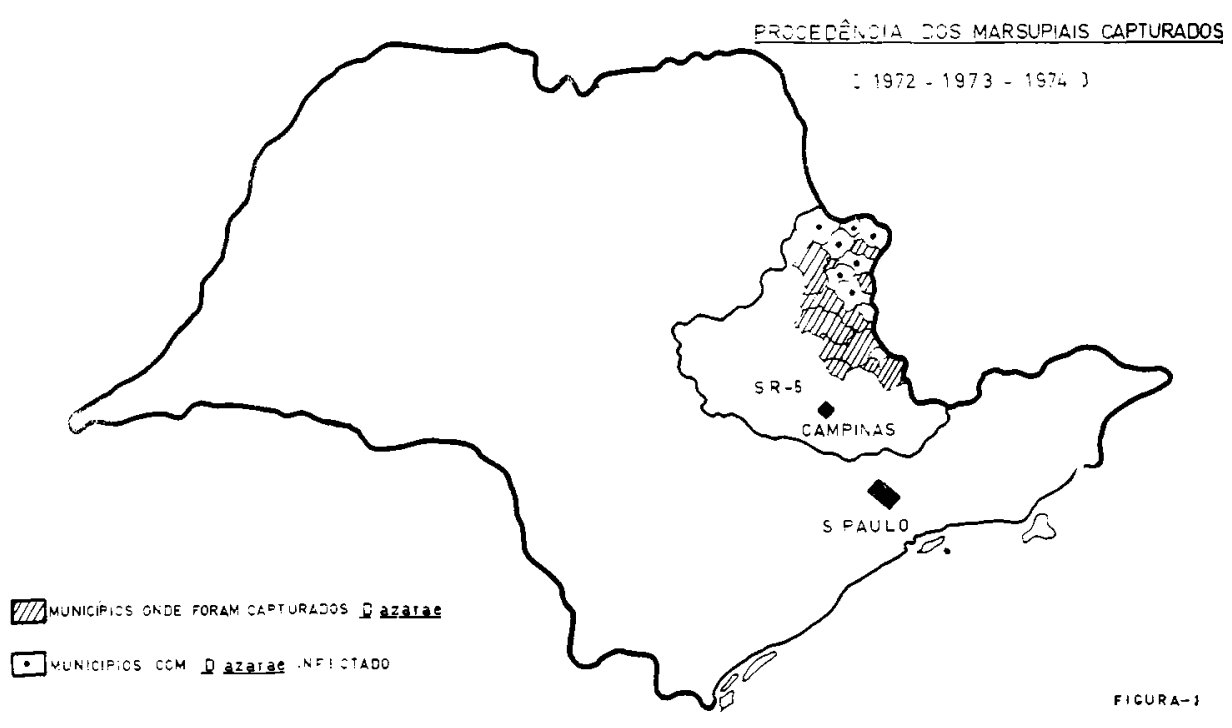


ROCHA E SILVA, E. O. et al. - Importância dos animaís sinantrópicos no controle da endemia chagásica. Rev. Saúde públ., S. Paulo, 9:371-81, 1975.

TABEL 1

Didelphis azarae - Local de captura e positividade para Trypanosoma tipo cruzi $(1972-1973-1974)$

\begin{tabular}{|c|c|c|c|c|c|c|c|c|c|}
\hline \multirow{3}{*}{ Munici- } & \multirow{3}{*}{$\underbrace{\begin{array}{c}\text { Local de } \\
\text { captura }\end{array}}_{\substack{\text { Post- } \\
\text { tivi- } \\
\text { dade }}}$} & \multicolumn{4}{|c|}{ No intradomícilio } & \multirow{2}{*}{\multicolumn{2}{|c|}{$\begin{array}{c}\text { Em diversos } \\
\text { tipos de anexos }\end{array}$}} & \multirow{2}{*}{\multicolumn{2}{|c|}{$\begin{array}{l}\text { Focos } \\
\text { periféricos } \\
\text { às casas }\end{array}$}} \\
\hline & & \multicolumn{2}{|c|}{ Casa habitada } & \multicolumn{2}{|c|}{ Casa desabitada } & & & & \\
\hline & & $\begin{array}{c}+T . \\
\text { tipo } \\
\text { cruzi }\end{array}$ & $\begin{array}{l}\text { Total } \\
\text { de exa- } \\
\text { mina- } \\
\text { dos }\end{array}$ & $\begin{array}{c}+T . \\
\text { tipo } \\
\text { oruzi }\end{array}$ & $\begin{array}{l}\text { Total } \\
\text { de exa- } \\
\text { mina- } \\
\text { dos }\end{array}$ & $\begin{array}{c}+T . \\
\text { tipo } \\
\text { cruzi }\end{array}$ & $\begin{array}{c}\text { Total } \\
\text { de exa- } \\
\text { mina- } \\
\text { dos }\end{array}$ & $\begin{array}{c}+T . \\
\text { tipo } \\
\text { cruzi }\end{array}$ & $\begin{array}{c}\text { Total } \\
\text { de exa- } \\
\text { mina- } \\
\text { dos }\end{array}$ \\
\hline
\end{tabular}

Aguai

Águas da Prata

Casa Branca

Caconde

Divinolândia

Itapira

Itobi

Lindóia

Mococa

Moji-Guaçu

Moji-Mirim

Pinhal

Santo Atonio da Posse

Soão João da Boa Vista

Săo José do Río Pardo

São Sebastião da Grama

Socorro

Tapiratiba

Vargem Grande do Sul

Total: 258 exemplares

(Positividade: $18,6 \%$ )

\begin{tabular}{|c|c|}
\hline- & - \\
\hline - & - \\
\hline- & \\
\hline - & - \\
\hline - & \\
\hline- & - \\
\hline - & $\bar{z}$ \\
\hline $\bar{z}$ & $\bar{z}$ \\
\hline- & - \\
\hline 一 & - \\
\hline 二 & $\bar{z}$ \\
\hline 2 & 4 \\
\hline 2 & 3 \\
\hline- & - \\
\hline- & - \\
\hline $\bar{z}$ & $=$ \\
\hline 4 & $\begin{array}{c}7 \\
(2,6 \%)\end{array}$ \\
\hline
\end{tabular}

$\bar{Z}$
$=$
$\bar{Z}$
$\bar{Z}$
$\bar{Z}$
$\overline{6}$
$\overline{3}$
$\overline{3}$

3

$3 \quad-\overline{1} \quad-\quad \overline{2}$

$\begin{array}{lllll}\overline{9} & \overline{1} & 7 & - & 1 \\ & & 7 & \end{array}$

$-$

3

-

$-$

$=$

-

1
4

$\frac{2}{1}$

二 $=\frac{1}{1}$

$=$

- 73

28

$\begin{array}{rrr}4 & 2 & 10 \\ 2 & - & 1\end{array}$

$\begin{array}{rrrr}2 & 10 & - & -\end{array}$

9

106

16

16

1

13

111

$13 \quad 34$

T A B E L 2

Inoculaçóes em camundongos, de cepas isoladas através de xenodiagnósticos realizados em D. azarae (1973 - 1974)

\begin{tabular}{|c|c|c|c|}
\hline $\begin{array}{r}\text { Inoculaçöes em } \\
\text { camundongos }\end{array}$ & & \multicolumn{2}{|c|}{ Resultado } \\
\hline $\begin{array}{l}\text { procedências dos } \\
\text { gambás xenados }\end{array}$ & inoculaçoes & $\begin{array}{c}\text { Positiva para } \\
\text { T. cruzi }\end{array}$ & $\begin{array}{l}\text { Negativa para } \\
\text { T. cruzi }\end{array}$ \\
\hline
\end{tabular}

\section{Caconde}

São João da Boa Vista

São José do Rio Pardo

Sāo Sebastião da Grama

Tapiratiba

Vargem Grande do Sul
1

$1 \overrightarrow{2}$

3

1

1

5

$\begin{array}{rr}- & 1 \\ - & 12 \\ 1 & 2 \\ -1 & - \\ 4 & 1\end{array}$

Total

23

6

$(26,0 \%)$

17

$(74,0 \%)$ 
ROCHA E SILVA, E. O. et al. - Importância dos animais sinantrópicos no controle da endemta chagásica. Rev. Saúde públ., S. Paulo, 9:371-81, 1975.

T A B E L A 3

Rattus rattus - Local de captura e positividade para Trypanosoma tipo cruzi $(1972-1973-1974)$

\begin{tabular}{|c|c|c|c|c|c|c|c|c|}
\hline \multirow[b]{3}{*}{$\begin{array}{l}\text { Munici- } \\
\text { pio de pro- } \\
\text { cedencia }\end{array}$} & \multicolumn{4}{|c|}{ No intradomicilio } & \multirow{2}{*}{\multicolumn{2}{|c|}{$\begin{array}{c}\text { Em diversos } \\
\text { tipos de anexos }\end{array}$}} & \multirow{2}{*}{\multicolumn{2}{|c|}{$\begin{array}{c}\text { Focos } \\
\text { periféricos } \\
\text { às casas }\end{array}$}} \\
\hline & \multicolumn{2}{|c|}{ Casa habitada } & \multicolumn{2}{|c|}{ Casa desabitada } & & & & \\
\hline & $\begin{array}{l}+T \\
\text { tipo } \\
\text { cruzi }\end{array}$ & $\begin{array}{c}\text { Total } \\
\text { de exa- } \\
\text { mins- } \\
\text { dos }\end{array}$ & $\begin{array}{l}+T \\
\text { tipo } \\
\text { cruzi }\end{array}$ & $\begin{array}{c}\text { Total } \\
\text { de exa- } \\
\text { mina- } \\
\text { dos }\end{array}$ & $\begin{array}{l}+T . \\
\text { tipo } \\
\text { cruzi }\end{array}$ & $\begin{array}{c}\text { Total } \\
\text { de exa- } \\
\text { mina- } \\
\text { dos }\end{array}$ & $\begin{array}{l}+T . \\
\text { tipo } \\
\text { oruzi }\end{array}$ & $\begin{array}{c}\text { Total } \\
\text { de exa- } \\
\text { mina- } \\
\text { dos }\end{array}$ \\
\hline Mococa & 一 & - & - & 一 & 一 & 3 & 一 & - \\
\hline Moj1-Guaçu & - & - & - & 一 & - & 1 & 一 & - \\
\hline Săo João da Boa Vista & 一 & 一 & 2 & 11 & - & 21 & - & - \\
\hline Săo José do Rio Pardo & 一 & 一 & - & - & - & 5 & - & - \\
\hline Săo Sebastião da Grama & $\longrightarrow$ & 一 & - & - & - & 2 & - & - \\
\hline Vargem Grande do Sul & - & - & - & 1 & 一 & 4 & - & - \\
\hline $\begin{array}{c}\text { Total: } 48 \\
\text { (Positividade: } 4,1 \% \text { ) }\end{array}$ & 一 & - & 2 & $\begin{array}{c}12 \\
(25,0 \%)\end{array}$ & 一 & $\begin{array}{c}36 \\
(75,0 \%)\end{array}$ & $\rightarrow$ & 一 \\
\hline
\end{tabular}

dos na região de Ribeirão Preto, contígua à região por nós trabalhada - e por aqueles por nós alcançados, podemos estimar a importância desse reservatório na região nordeste do Estado, em que pese o tempo decorrido entre ambos. A positividade de $18,64 \%$ para os 258 exemplares de $D$. azarae que examinamos, não fica longe dos $21,43 \%$ encontrados para os 126 exemplares da mesma espécie, por eles examinados. Acrescente-se que o teste estatístico de proporções não mostrou diferenças significativas entre essses valores.

Dos $23 D$. azarae cujo sangue serviu de repasto a exemplares do $T$. infestans em laboratório, determinando o aparecimento de formas tripomastigotas no tubo intestinal dos triatomíneos, 11 deles foram capturados no interior de moradias. Dessas, três encontravam-se habitadas na ocasião da captura dos marsupiais. Numa delas, deve ser assinalado que, além do gambá, foram capturados também exemplres do
Panstrongylus megistus. $\mathrm{O}$ fato salienta a necessidade de manutenção das atividades de controle em áreas limpas do $T$. infestans, mas que continuam a apresentar junto ao homem exemplares de espécies outras. A presença de triatomíneos ditos semi-domiciliários nas casas, concomitante com a presença dos reservatórios sinantrópicos, poderá propiciar o reaparecimento a qualquer momento da cadeia domiciliar da doença de Chagas. Nestas circunstâncias, resta como recurso imediato do controle, a procura periódica dos transmissores, completada com a captura de reservatórios nẩo domésticos, realizada no interior das casas, nos anexos e adjacências.

Se, por um lado, é evidente que o reaparecimento da cadeia domiciliar não inclui obrigatoriamente o homem, por outro lado, é verdade também que haverá possibilidade da ocorrência de uma exacerbação de virulência da cepa, na transferência de reservatório. Fato que torna po- 
ROCHA E SILVA, E. O, et al. - Importancia dos animais sinantrópicos no controle da endemia chagásica. Rer. Saúde pübl., S. Paulo, 9:371-81, 1975.

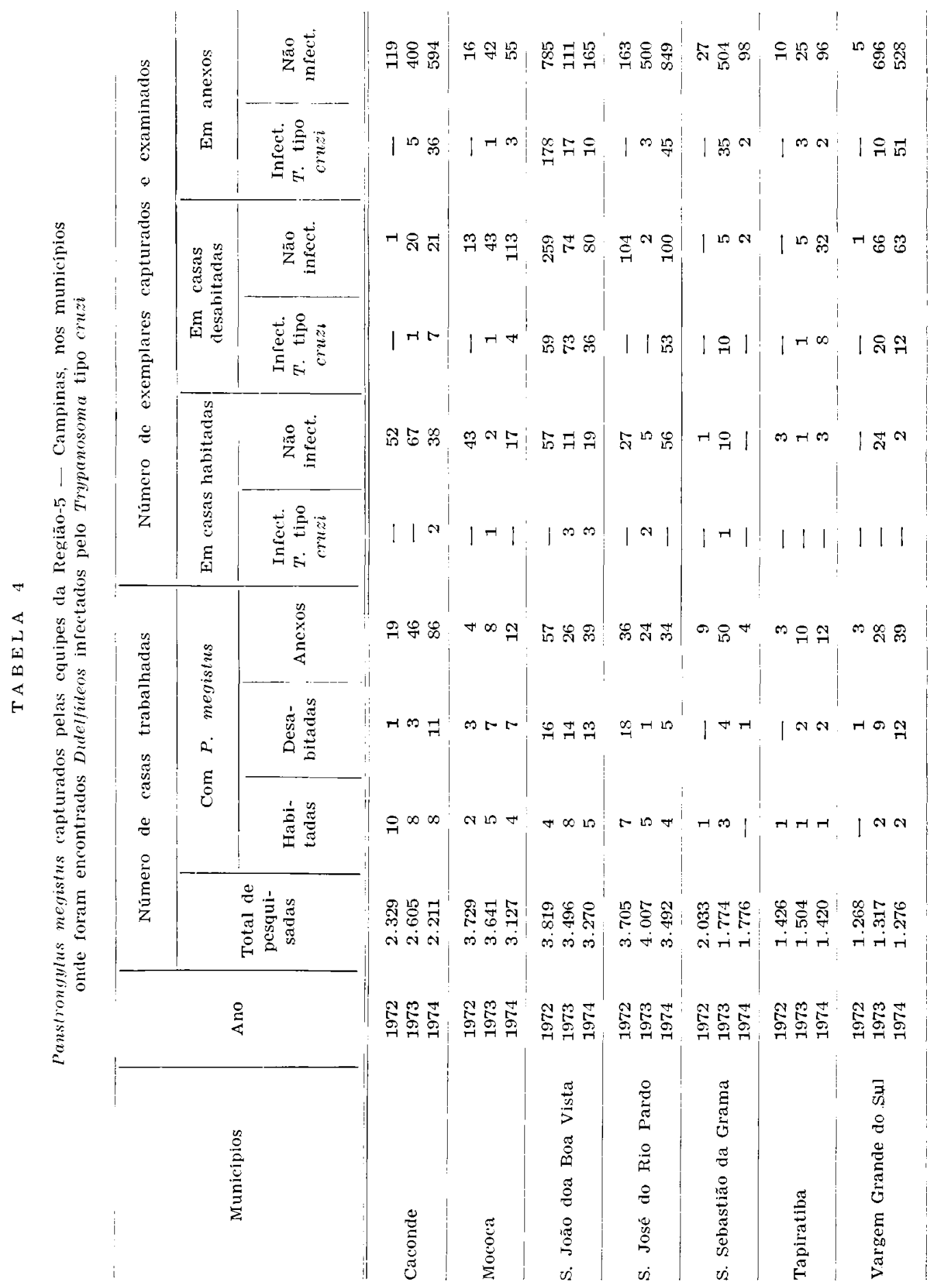


ROCHA E SILVA, E. O. et al. - Importância dos animais sinantrópicos no controle da endemia chagásica. Rev. Saúde pübl., S. Paulo, 9:371-81, 1975.

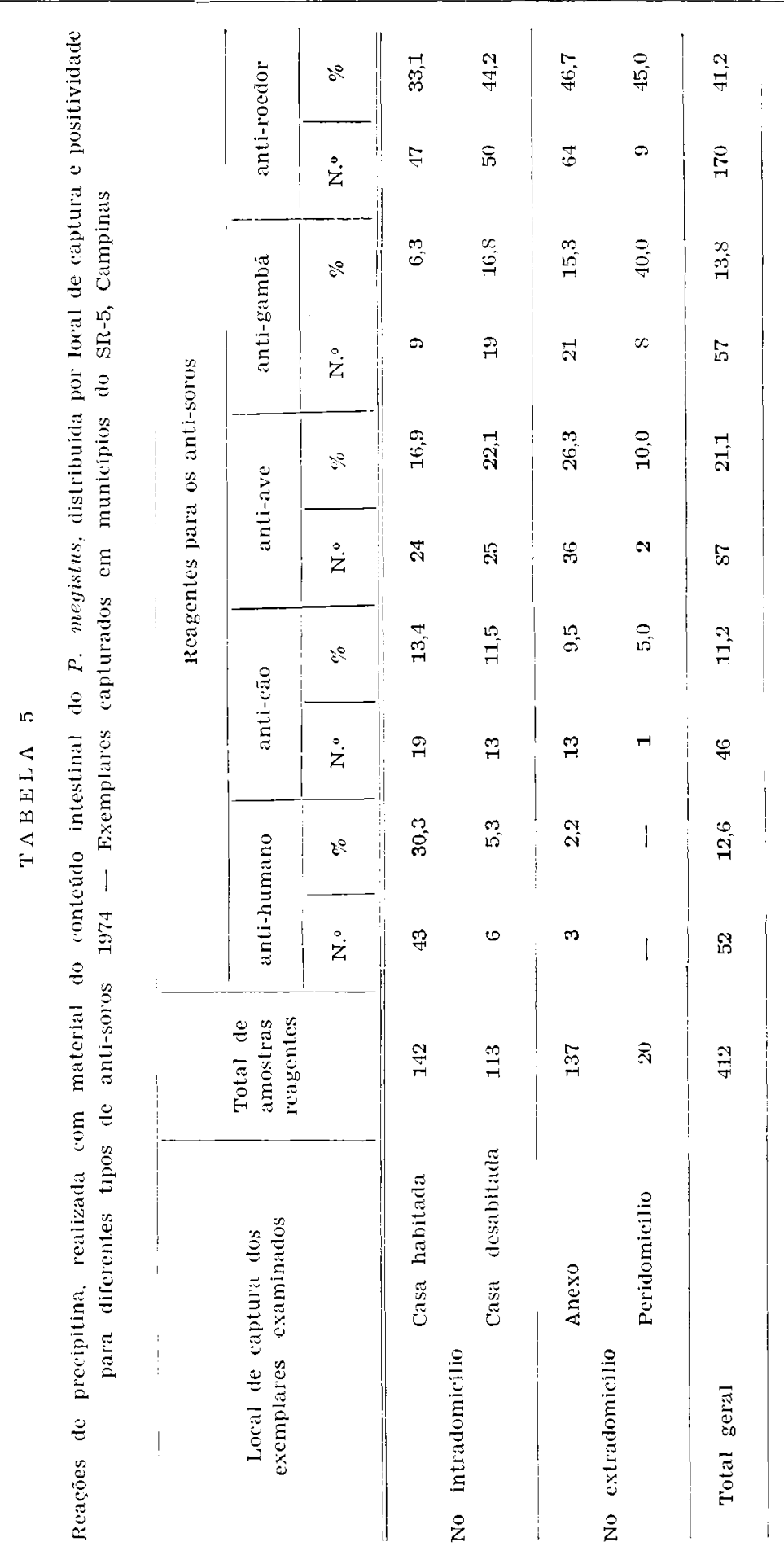


ROCHA E SILVA, E. O. et al. - Importância dos animais sinantrópicos no controle da endemia chagásica. Rev. Saúde públ., S. Paulo, 9:371-81, 1975.

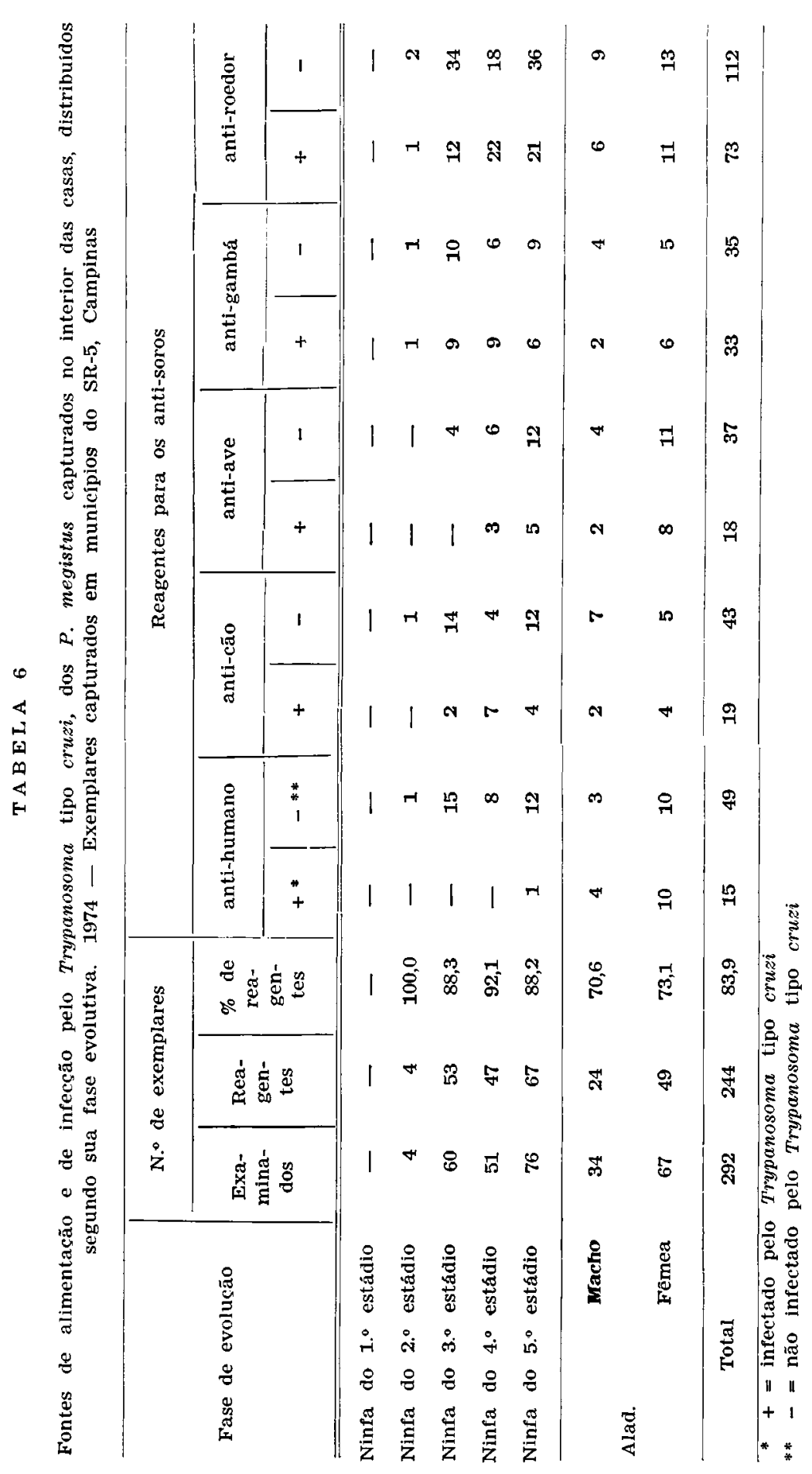


ROCHA E SILVA, E. O. et ai. - Importância dos animais sinantrópicos no controle da endemia chagásica. Rev. Saúde públ., S. Paulo, 9:371-81, 1975.

tencialmente perigosa a presença do $T$. cruzi junto ao homem, mesmo sem atingi-lo de imediato. Os dados da Tabela 4 , parecem-nos suficientes para demonstrar a potencialidade sugerida.

Das 23 amostras isoladas através de xenodiagnóstico e inoculadas, apenas 6 infectaram camundongos jovens, sendo que dessas, 4 foram isoladas de $D$. azarae capturados no município de Vargem Grande do Sul, e as restantes, de gambás procedentes dos municípios de São Sebastião da Grama e São José do Rio Pardo, próximos do anterior. Este fato deixa em aberto, por falta de adequados elementos de identificação, a possibilidade de existir, na área trabalhada, outros tripanossomos que não o $T$. cruzi.

No que se refere aos 48 exemplares de Rattus rattus examinados (Tabela 3 ), observou-se que $8(19,51 \%)$ ao exame do sangue periférico, mostraram-se infectados pelo $T$. tipo lewisi e através do xenodiagnóstico, 2 infectados pelo $T$. tipo cruzi $(4,13 \%)$.

Interessa pois às campanhas de controle, o reconhecimento correto das formas de tripanossomos semelhantes ao $T$. cruzi, detectados nos reservatórios encontrados nas proximidades do homem. Por isso, essas campanhas deveräo considerar de valor, atividades de laboratório que visem, sempre que possvel, esclarecer a natureza dos flagelados encontrados.

As considerações acima e as atividades desenvolvidas na região de Campinas, no que se refere a capturas de reservatórios não domésticos, estão perfeitamente enquadradas nas recomendações da Organização Panamericana da Saúde ${ }^{5}$ (1970): "És importante que los reservorios no domesticos sean reconocidos e identificados. Sin embargo, para restabelecer su verdadero papel de reservorios de la infeccion humana, los tripanossomos que en ellos se encuentrem deben ser cuidadosamente estudiados".

A Tabela 5 apresenta os dados relativos às fontes de alimentos dos exemplares do $P$. megistus, em função do local de captura: casa habitada, casa desabitada, construções anexas ou periferia das moradais. Esses dados sugerem que independentemente do local da captura, os roedores apresentam-se como importante fonte de alimento desses triatomíneos, na área considerada. Em proporção menor, o mesmo ocorre com os marsupiais. As aves, em qualquer situação, apresentam-se como alternativa alimentar. Com referência às reações para soros, anti-sangue humano e anti-cão, observa-se na Tabela 5 expressiva positividade de ambos nas casas e o último, também nos anexos.

E importante esclarecer que os dados contidos nessa Tabela, não constituem o resultado dos exames realizados com a totalidade dos exemplares testados, mas apenas com um elemento representativo de cada tipo de alimentação, encontrado nos focos estudados. Procuramos desse modo evitar desvios provocados pela presença de numerosos exemplares, capturados no mesmo local utilizando a mesma fonte alimentar.

Saliente-se que os números dessa Tabela, embora modestos, são sugestivos de que o $P$. megistus na região nordeste do Estado de São Paulo, apresenta comportamento que possibilita sua penetração nas casas, encontrando no domicílio não apenas abrigo, mas também alimento e, em consequêencia, perspectivas de colonização. Isso só não tem ocorrido com maior intensidade, ou melhor dizendo, os focos encontrados nas casas só não apresentam elevado número de exemplares, devido ao trabalho anual de busca e captura, realizado pelas equipes de campo da SUSAM.

A Tabela 6 apresenta os resultados dos testes realizados com todos os exemplares, capturados no interior de casa habitadas ou não, distribuídos de acordo com sua fase de evolução e considerando-se a presença ou não do $T$. cruzi, na luz intesti- 
ROCHA E SILVA, E. O, et al. - Importância dos animais sinantrópicos no controle da endemia chagásica. Rev. Saúde puibl., S. Paulo, 9:371-81, 1975.

nal. Os dados assim distribuídos sugerem que, dentro dos limites da nossa observação, roedores e marsupiais não só constituem importantes fontes alimentares do P. megistus na área, como suas principais fontes de infecção pelo $T$. cruzi, notadamente os didelfídeos. Esta impressão decorre do número de ninfas de estádios jovens encontradas positivas, concomitantemente, para o sangue desses animais e para o $T$. tipo cruzi.

\section{CONCLUSŌES}

1. Em diversos municípios da Regional-5 - Campinas, vertebrados dos gêneros Didelphis e Rattus rattus se comportam como reservatórios do Trypanosoma tipo $c r u z i$, sendo encontrados com freqüência nas casas habitadas e sobretudo desabitadas, nos seus prédios anexos e áreas periféricas. Juntamente com esses reservatórios, têm sido capturados exemplares do Panstrongylus megistus infectados. Estes achados ressaltam a possibilidade da reintrodução na área, do ciclo domiciliar da doença de Chagas com a participação do homem.
2. Em conseqüência, as atividades de controle da endemia nessa região, apesar da ausência do seu principal vetor (Triatoma infestans), prosseguem, incluindo além da periódica busca dos transmissores e indispensável expurgo dos focos, a captura de animais sinatrópicos, notadamente didelphídeos.

3. As campanhas de controle deverão contar com laboratórios em condições de identificar, na medida do possível, os tripanossomos encontrados nos vetores e reservatórios e não simplesmente dedectar formas flageladas nas fezes dos triatomíneos.

4. Deverão também desenvolver atividades de avaliação e pesquisa, capazes de fornecer subsídios ao andamento dos trabalhos e aprimorar métodos de controle.

\section{A GRADECIMENTOS}

Aos senhores Antenor do Nascimento Ferraz Filho, José de Campos Camargo e Lourival Sargaço, auxiliares técnicos, pela colaboração ao trabalho.

RSPU-B/276

Rocha 'E '́llva, E. O. et al. - [On the importance of Synantropic animals in the control of Chagas' disease (endemic)]. Rev. Saúde públ., S. Paulo, 9:371-81, 1975 .

SUMMARY: Emphasis is layed upon the fact that in some areas, the routine methods used to control vectors of Chagas' disease, must be complemented by seizure activities of $\mathrm{T}$. cruzi reservoirs, specially marsupials (Didelphis) and rodents (Rattus). A study was undertaken in some municipal districts of the Administrative Region of Campinas, State of S. Paulo, where the presence of reservoirs and peridomestic triatomines (Panstrongylus megistus) allow favourable conditions for the reintroduction of the domiciliary parasitic cycle. The results of the search of Trypanosoma type cruzi in these triatomines and the mentioned reservoirs are commented. Their association with the precipitin reaction in order to allow detection of the type of blood ingested by the P. megistus captured in the houses was studied.

Uniterms: Trypanosomiasis, South American. Panstrongilus megistus. Trypanosoma cruzi. 
ROCHA E SILVA, E. O. et al. - Importância dos animais sinantrópicos no controle da endemia chagásica. Rev. Saúde públ., S. Paulo, 9:371-81, 1975.

\section{REFERENCIAS BIBLIOGRAFICAS}

1. BARRETO, M. P. et al. - Estudos sobre reservatórios e vetores silvestres do Trypanosoma cruzi. VII. Investigações sobre a infeç̧ão natural de gambás por tripanossomos semelhantes ao T. cruzi. Rev. bras. Biol., Rio de Janeiro, 24:289-300, 1964.

2. BARRETO, M. P. - Tripanossomos semelhantes ao Trypanosoma cruzi em animais silvestres e sua identificação com o agente etiológico da doença de Chagas. Rev. Inst. Med. trop., S. Paulo, 7:305-15, 1965.

3. FORATTINI, O. P. et al. - Aspectos ecológicos da Tripanossomose Americana. I. Observações sobre Panstrongylus megistus e suas relaçōes com fo$\cos$ naturais da infecção, em área urbana da cidade de São Paulo, Brasil. Rev. Saúde públ., S. Paulo, 4: 19-30, 1970.

4. FORATtinI, O, P. et al. - Aspectos ecológicos da Tripanossomose Americana. II. Distribuição e dispersão local de triatomíneos em ecótopos naturais e artificiais. Rev. Saude públ., s. Paulo, 5:163-91, 1971.

5. ORGANIZACION Panamericana de la Salud - Grupo de Estudio sobre la Enfermidad de Chagas, Washington,
D.C., 1969. Informe. Washington, D.C., 1970. (Publicações Cientificas, 195). p. 10-2.

6. RIBEIRO, R. D. \& JUAREZ, E. - Infecção natural do rato. Oryzomys nigripes (Desmarest, 1819) pelo T. cruzi. Rev. Soc. bras. Med. trop., 6:11-8, 1972 .

7. ROCHA E SILVA, E. O. et al. - Consideraçōes sobre alguns tripanosomatideos, presentes no tubo intestinal de triatomíneos. Apresentado no XI Congresso da Sociedade Brasileira de $\mathrm{Me}$ dicina Tropical, Rio de Janeiro, 1975.

8. SIQUEIRA, A. F. - Estudos sobre a reação de precipitina aplicada na identificação do sangue ingerido pelos triatomineos. Rev. Inst. Med. trop., S. Paulo, 2:41-53, 1960.

9. ZELEDON, R. - Epidemlology, mode of transmission and reservoir of Chagas' disease. In: Symposium on Trypanosomiasis and Leishmaniasis with special reference to Chagas'disease, Caracas, Amsterdam, Excerpta Medica Foundation, 1974 [Ciba Foundation Symposium 20 (new series)] p. 51-71.

Recebido para publicasão em 14-03-75 Aprovado para publicação em $04-04-75$ 